\title{
Towards an optimal aiming for molten salt power towers
}

\author{
Robert Flesch ${ }^{\mathrm{a}}$, Cathy Frantz ${ }^{\mathrm{b}}$, Daniel Maldonado Quinto ${ }^{\mathrm{c}}$, Peter \\ Schwarzbözlc \\ ${ }^{a}$ Institute of Solar Research, German Aerospace Center, Professor-Rehm-Straße 1, \\ D-52428 Jülich, Germany \\ ${ }^{b}$ Institute of Solar Research, German Aerospace Center, Pfaffenwaldring 38, D-70569 \\ Stuttgart, Germany \\ ${ }^{c}$ Institute of Solar Research, German Aerospace Center, Linder Höhe, D-51147 Köln, \\ Germany
}

\begin{abstract}
Finding a suitable aiming strategy for receivers of power towers can be challenging, especially for receivers using molten salt as heat transfer fluid as the allowable flux density decreases dramatically with increasing salt temperature. In this paper a very fast, steady-state model for the molten salt receiver is presented. This model is combined with a ray-tracing software and a metaheuristic optimization procedure. The thermal model is used to calculate the actual temperature and mass flow in the receiver which are then used to calculate the operational limits for the flux density. It is demonstrated that such an optimized aiming strategy can outperform a parameter based aiming strategies by more than $2 \%$.
\end{abstract}

Keywords: concentrating solar power, molten salt receiver, aim point optimization, ant colony optimization

\section{Introduction}

One option to utilize solar energy for a renewable electricity production 3 are solar power towers using molten salt as heat transfer fluid in the receiver. 4 Mirrors reflect the sunlight to heat up the salt inside the receiver which is 5 located at the top of the central tower. The hot salt can be easily stored inside

Email address: robert.flesch@dlr.de (Robert Flesch) 
6

hot storage tanks. This allows a demand-oriented production of electricity. Therefore this technology is a promising option for future energy production. The flux density on the receiver surface is limited due to

- thermal stresses inside the tube caused by the one-sided radiation

- an upper temperature limit for the molten salt to avoid rapid corrosion of the tubes and degradation of the salt.

The resulting allowable flux density on the absorber tube surface is a key design and operational parameter for the receiver.

If all heliostats of the field aimed on the of the receiver or in case of a cylinder on the centerline respectively, almost all radiation would hit the receiver, but the maximum flux density would be beyond the limits. Therefore, the aim points of the heliostats are distributed over the receiver surface, which increases the fraction of radiation, which does not hit the receiver: the so-called spillage losses increase. This is the trade-off for the aiming strategy. An optimal aiming should maximize the power on the receiver surface with respect to the allowable flux density. In case of a receiver using molten salt as heat transfer fluid the situation gets even more complicated. The flux limit strongly depends on both temperature and mass flow of the salt. For this reason, the spatially distributed values of flux limits have to be updated continuously in accordance with the local salt temperatures and load situation.

\section{State-of-the-art}

A detailed analysis description of the flux limits and aiming strategy used in the molten salt tower demonstration project Solar Two can be found in Vant-Hull (2002). In this paper, Vant-Hull introduced the concept of the allowable flux density (AFD). In this concept, both limitations, stress and peak salt temperature directly at the wall, are transferred into a flux density limit as a function of local salt temperature and salt velocity. The resulting flux density limits are shown in Fig. 1. With higher salt temperatures the flux limit caused by the film temperature becomes the limiting factor, resulting in the kinks in the curves.

Furthermore, Vant-Hull describes the aiming strategy which was used in the Solar Two plant to keep the flux within the limits. The flux coming from each heliostats was approximated by a cone. The opening angle of the cone 


\begin{tabular}{|c|c|c|c|}
\hline \multicolumn{4}{|c|}{ Nomenclature } \\
\hline \multirow{4}{*}{$\begin{array}{l}\alpha \\
\Delta L \\
\epsilon \\
\theta\end{array}$} & Absorptance & \multirow{2}{*}{$f_{\sigma}$} & \multirow{2}{*}{$\begin{array}{l}\text { Extension factor for the as- } \\
\text { sumed beam cone angle }\end{array}$} \\
\hline & Length of the tube element & & \\
\hline & Emissivity & $h$ & Heat transfer coefficient tube \\
\hline & $\begin{array}{l}\text { Bulk salt temperature in de- } \\
\text { gree Fahrenheit }\end{array}$ & \multirow[t]{2}{*}{$h_{\text {conv }}$} & \multirow{2}{*}{$\begin{array}{l}\text { Convective heat transfer co- } \\
\text { efficient due to natural and } \\
\text { forced convection (wind) }\end{array}$} \\
\hline$v$ & Temperature in degree & & \\
\hline$\dot{m}$ & $\begin{array}{l}\text { Fahrenhelt } \\
\text { Mass flow }\end{array}$ & $k$ & $\begin{array}{l}\text { Thermal conductivity of the } \\
\text { tube material }\end{array}$ \\
\hline$\dot{q}_{\mathrm{F}}^{\prime \prime}$ & Flux density & \multirow[t]{2}{*}{$L F$} & \multirow{2}{*}{$\begin{array}{l}\text { Load factor: ratio of local } \\
\text { absorbed flux and allowable } \\
\text { flux density }\end{array}$} \\
\hline$\dot{q}_{\text {net }}^{\prime \prime}$ & $\begin{array}{l}\text { Net flux density on receiver } \\
\text { tube }\end{array}$ & & \\
\hline \multirow[t]{2}{*}{$\xi$} & \multirow{2}{*}{$\begin{array}{l}\text { Second parameter in aiming } \\
\text { strategy }\end{array}$} & $p$ & Penalty factor \\
\hline & & $r$ & Slant range \\
\hline$A_{\mathrm{p}}$ & Tube element projected area & $T_{\mathrm{Fc}}$ & Tube crown temperature \\
\hline$A F D$ & Allowable flux density & $T_{\mathrm{F}}$ & Front element temperature \\
\hline$c$ & Constant for penalty factor & $T_{\mathrm{Ic}}$ & Film crown temperature \\
\hline$c_{p}$ & Specific heat capacity of salt & $T_{\mathrm{S}}$ & Fluid temperature \\
\hline$d_{\mathrm{I}}$ & Inner diameter & $T_{\mathrm{inf}}$ & Ambient temperature \\
\hline$d_{\mathrm{O}}$ & Outer diameter & $v$ & Velocity of the salt in the \\
\hline$e$ & Exceedance of limits & & \\
\hline$f_{0}$ & $\begin{array}{l}\text { First parameter in aiming } \\
\text { strategy }\end{array}$ & $v_{\mathrm{dp}}$ & $\begin{array}{l}\text { Velocity of the salt in the } \\
\text { tubes for the design point }\end{array}$ \\
\hline
\end{tabular}

is chosen in a way that the cone envelopes a predefined amount of power of the Gaussian distributed flux density of a heliostat. Half of the heliostats aimed in a way that the assumed outer edge of their beam coincides with the upper rim of the receiver. The other half aimed on the lower part of the 


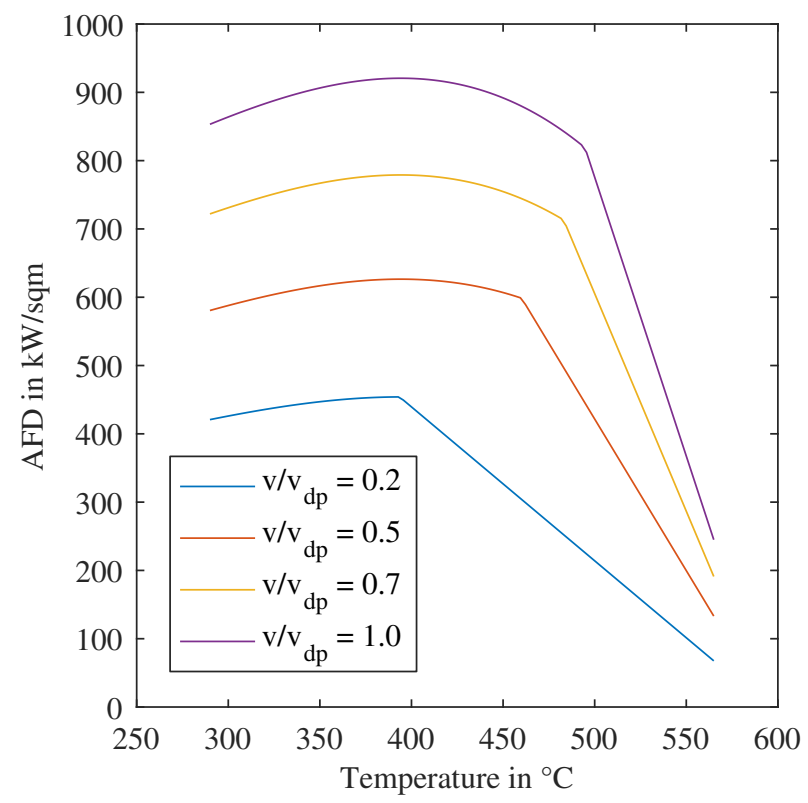

Figure 1: Flux density limits applied in the Solar Two plant as a function of velocity and local salt temperature

receiver in an analogous fashion. In order to compute the aiming points for each heliostat, a respective opening angle of the beam has to be deduced. This angle can be calculated based on sunshape, mirror and tracking error as well as astigmatism. The resulting opening angle is then multiplied with a constant factor; the aiming strategy parameter. During operation the flux was monitored by numerical simulations and heliostats causing excessive flux were removed from the receiver. Since removing heliostats reduces the power on the receiver and therefore also the velocity of the salt flow to maintain the desired salt outlet temperature level, the AFD is reduced as well. This might lead to additional areas with overflux and the defocussing of additional heliostats. Such instability was observed during the operation of Solar Two (Pacheco et al. (2002)).

For the plants having started operation during the past years no detailed information about the aiming strategy has been published. Nevertheless, in scientific literature methods and strategies for receivers using molten salt have been published. Sanchez-Gonzalez et al. (2016) developed a fit algorithm to optimize the aiming strategy for a molten salt receiver. A thermal model was used to calculate an AFD database including the limits by stresses 
and temperature. During optimization the mass flow and local temperatures were calculated and the actual flux density was compared to the database values of AFD. The aiming strategy followed the idea of Vant-Hull, but instead of using one factor for the whole field, individual factors for small groups of heliostats were used. These factors were determined during an optimization procedure.

Beside the presented methods for molten salt receivers several analyses of aim point optimization for other receiver types were published. Salom et al. (2013) and Besarati et al. (2014) used optimization algorithms to create flux density distribution as homogeneous as possible. Belhomme et al. (2014) used an adapted ant colony algorithm to maximize the output of a concentrated photo-voltaic receiver. García-Martín et al. (1999) developed a heuristic knowledge-based heliostat control strategy for the open volumetric receiver at the Plataforma Solar de Almería.

\section{Methods}

\subsection{Parameter based aiming}

Beside the aim point optimization methodology, which will be described in the next section, a newly developed parameter based aiming strategy is presented, because the Vant-Hull aiming strategy might fail to produce a suitable flux density distribution. The Vant-Hull strategy is based on one single factor: this factor increases the cone angle of each heliostat from its theoretical minimum given by an assumed beam error. With increasing factor the cone angle increases and the cone will envelope a higher amount of the radiation of the heliostat. Therefore a high factor value leads to large angle for the cones. As a result the aim points of the heliostats lie close to the receiver centerline and cause a peak in the flux density at the receiver centerline. Decreasing this factor causes the heliostats to aim more towards the edges of the receiver. In case of heliostats with good beam quality this might cause so-called shoulders at the edges of the receiver, in which the limits are exceeded as well. To overcome this issue, the so-called modified Vant-Hull aiming strategy is developed. In this method the factor for the cone angle $f_{\sigma}$ is calculated by

$$
f_{\sigma}=f_{0}+\xi \cdot r
$$

with the two parameters $f_{0}$ and $\xi$ and slant range of heliostat and center of the receiver $r$. When using $\xi=0$ the aim strategy equals the conventional 
Vant-Hull aiming. With $\xi>0$, the first parameter $f_{0}$ gives a possibility to limit the amount of spillage due to the inaccuracy of the analytical image size computation: it defines the minimum distance between aiming point and receiver edge as a function of the computed image size. The second parameter $\xi$ introduces the possibility to scale the punishment for the distance between heliostat and receiver. This allows an even distribution of the flux over the receiver surface, also for heliostats with small beam errors. This parameter based aiming strategy will be used as reference for the aim point optimization.

\subsection{Aim point optimization}

In the current analysis the methodology and tools described in Belhomme et al. (2014) are adapted to be used for a molten salt receiver. In the following of this section the approach for the optical simulation and the optimization approach is described very briefly. Subsequently, the newly developed thermal model for the receiver is discussed in detail. The sequence of steps in the optimization procedure is shown in Fig. 2.

\subsubsection{Optical model and optimization procedure}

Since the approach of the optimization procedure has already been described in Belhomme et al. (2014) in detail, it is only summarized in this section. The flux density distribution is calculated using the software tool STRAL (Belhomme et al. (2009)), which has been developed at the German Aerospace Center. The tool offers the option to include measured surface data of heliostats and therefore, it is capable to calculate a very accurate prediction of the resulting flux on the receiver surface.

By using a discretized grid of aim points the optimization problem is transferred into a combinatorial problem. Therefore, algorithms like the ant colony algorithm can be applied to find an optimal solution.

The usage of a ray tracing tool for the calculation of the flux density distribution has the advantage of its high accuracy, but the drawback of the large computational effort. To overcome this issue, the image of each heliostat is calculated and stored for each aim point before starting the optimization (precalculation step). During precalculation of the flux distribution of a single heliostat, all other heliostats aim on a predefined aim point. This introduces a slight inaccuracy since the shading and blocking depends on the orientation of the other heliostats. However, the changes in orientation of the heliostats and thus the inaccuracy are very small and the gain in computational speed is immense. Therefore this approach is chosen. By using a 


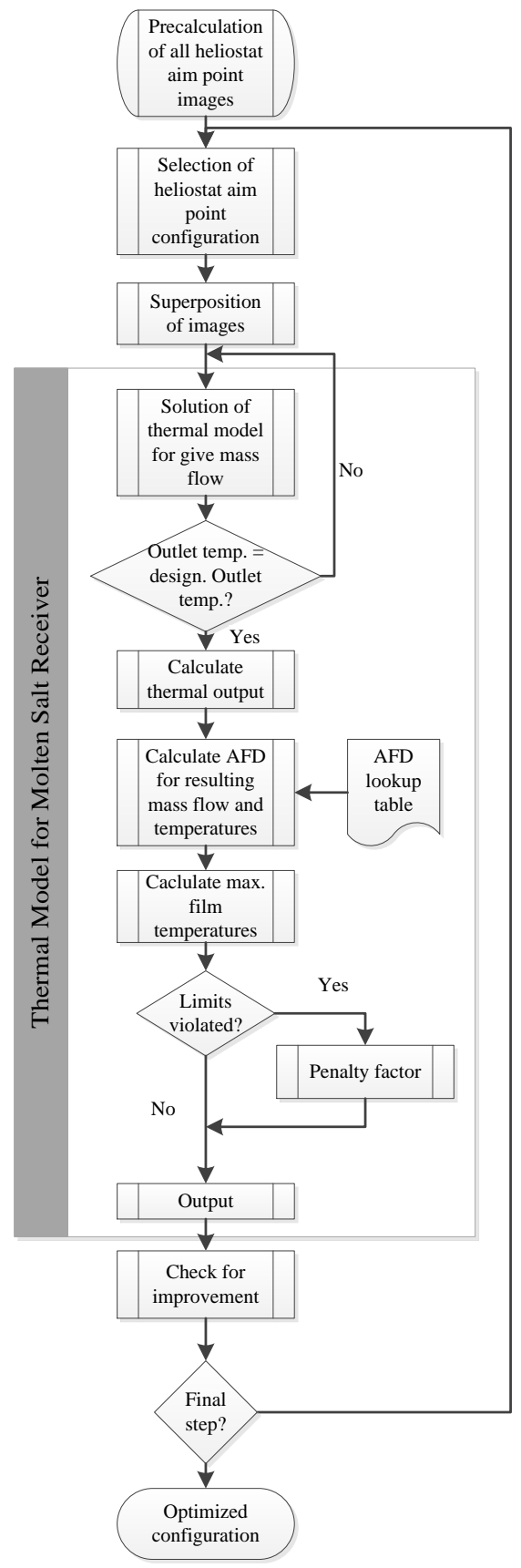

Figure 2: Flow scheme of the optimization procedure 


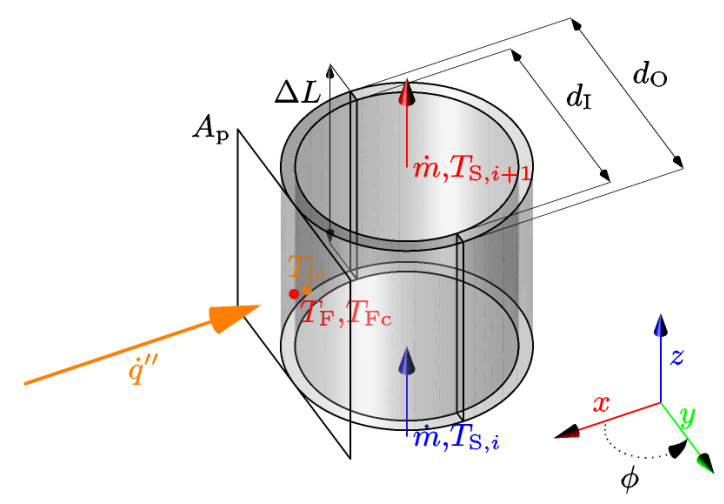

Figure 3: Depiction of one element of a single tube used for the thermal modeling

fixed number of aim points with fixed positions the optimization problem is transferred into a combinatorial problem and algorithms like the ant colony algorithm can be applied to find an optimal solution with heuristic informations. Namely the global quality of the current solution, here the receiver performance for the overall aim point configuration, and the local quality of a discrete change in the aim point assignment for a single heliostat, here the intercepted power of one heliostat. Belhomme et al. (2014) transferred the ant colony optimization metaheuristic to the aim point optimization problem by defining the aim point configuration as the trail of an ant discovering a forage source. Every aim point heliostat assignment is a part of this trail. The receiver performance as the global quality value corresponds to the length of the overall trail. The intercepted power of one heliostat as the local quality value corresponds to the myopic information of a single ant while discovering the trail. During the optimization process the current aim point configuration is improved according the heuristic informations. Starting point is a sub-optimal aim point configuration. In each optimization step all corresponding precalculated images are superposed to calculate the resulting flux density distribution. The resulting flux density distribution is used by the receiver model, which is described in the following section, to calculate one single output value.

\subsubsection{Thermal model}

In general, the receivers used in solar power towers consist of several panels. The salt passes the panels sequentially. Each panel is composed of several parallel tubes. In the following we describe the approach which was 
chosen to obtain a fast model as it is required for the optimization. The model calculates the relevant local states of the salt flow (mass flow and temperatures) required to calculate the AFD. To obtain a fast model several assumptions are made:

- The radiative heat exchange between the tubes (reflection and emission) is neglected. Neighboring tubes are exposed to almost the same flux and the temperatures are similar (except the for the neighboring tubes belonging to different panels). Therefore, the thermal radiation from one tube to its neighbors equals approximately the radiation coming back from the neighboring tubes. If the radiation exchange is neglected, all tubes of a panel can be treated individually.

- The heat conduction along the tube axis inside the tube material is neglected. The influence is assumed to be small as the tubes are very thin.

- The tubes are discretized in two elements in circumferential direction: one element for the front side and one for the backside. We assume that no heat is conducted from the front element to the back element which implies that the total absorbed heat flux is transported radially through the front element.

- For the flux coming from the field and the radiation exchange with the environment the front element is treated like a flat plate with a surface area corresponding to the projected area of the tube.

- The total mass flow in one panel is equally split between all absorber tubes.

- The outlet temperature of a panel is equal to the mixing temperature of the outlet temperatures of its tubes. The assumption implies adiabatic headers. This outlet temperature is used as the inlet temperature for the downstream panel.

The axial discretization can be chosen by the user. The different physical values of one tube element are depicted in Fig. 3. Additionally, the user can specify whether each tube of a panel should be simulated or just a userspecified number of representative tubes. 
Applying the previously described assumptions we can express the heat which is transferred through the tube $\dot{Q}_{\text {cond }}$ with

$$
\dot{Q}_{\text {cond }}=\alpha A_{\mathrm{p}} \dot{q}_{\mathrm{F}}^{\prime \prime}-\sigma \epsilon A_{\mathrm{p}} \cdot\left(T_{\mathrm{F}}^{4}-T_{\mathrm{inf}}^{4}\right)-h_{\mathrm{conv}} A_{\mathrm{p}}\left(T_{\mathrm{F}}-T_{\mathrm{inf}}\right)
$$

using the absorptance $\alpha$, the emissivity $\epsilon$, the projected area $A_{\mathrm{p}}$, the flux density from the field $\dot{q}_{\mathrm{F}}^{\prime \prime}$, the convective coefficient $h_{\mathrm{conv}}$ and the front and ambient temperature $T_{\mathrm{F}}$ and $T_{\mathrm{inf}}$ respectively. The heat flux $\dot{Q}_{\text {cond }}$ has to be transported through the tube material into the fluid. The heat transfer to the fluid is modeled with a one dimensional approach and with a corrlation based calulation of the convective heat transfer coefficient. For the heat transport through tube and to the fluid the following equation is used

$$
\dot{Q}_{\text {cond }}=\frac{1}{\frac{1}{h \frac{\pi}{2} d_{\mathrm{I}} \cdot \Delta L}+\frac{1}{\pi k \Delta L} \cdot \ln \left(\frac{d_{\mathrm{O}}}{d_{\mathrm{I}}}\right)} \cdot\left(T_{\mathrm{F}}-T_{\mathrm{S}, i+1}\right) .
$$

In this equation we have used the symbols $h$, for the convective heat transfer inside the tube, $k$ for the thermal conductivity of the tube material, $d_{\mathrm{I}}$ and $d_{\mathrm{O}}$ for the inner and outer diameter of the tube, $\Delta L$ for the length of the tube element and $T_{\mathrm{S}, i+1}$ for the temperature of the fluid. Finally, the heat transferred to the fluid $\dot{Q}_{\text {cond }}$ leads to a temperature rise

$$
\dot{Q}_{\text {cond }}=\dot{m} c_{p}\left(T_{\mathrm{S}, i+1}-T_{\mathrm{S}, i}\right)
$$

from the inlet temperature $T_{\mathrm{S}, i}$ to the outlet temperature $T_{\mathrm{S}, i+1}$ with the mass flow of salt in the tube $\dot{m}$ and the specific heat capacity $c_{p}$.

By combining the three equations 2,3 and 4 with the variables $\dot{Q}_{\text {cond }}$, $T_{\mathrm{F}}$ and $T_{\mathrm{S}, i+1}$ for a given mass flow $\dot{m}$ and inlet temperature $T_{\mathrm{S}, i}$ we obtain a nonlinear equation e.g. for the front temperature. If the influence of the temperature on the properties like $k$ and on those in the calculation of $h$ is neglected, this equation can be solved analytically. As a first guess all properties depending on temperatures are evaluated for the known inlet temperature. The solution which is obtained with this assumption is refined in a second iteration, in which all properties are evaluated for the temperatures of the initial solution. In a real plant the mass flow is adjusted by the control system to obtain a predefined outlet temperature. For this reason, the mass flow is not known prior to the solution. In the model an iterative approach is used for the calculation of the mass flow. An initial estimate of the mass flow is calculated by using the gross flux and a guess of the receiver 
efficiency. For this mass flow all temperatures are calculated sequentially, leading to an outlet temperature. In the following iterations the mass flow is adjusted until the outlet temperature is close enough to the given outlet temperature.

Solving the thermal model gives the mass flow of salt and the local salt temperatures for the given flux density distribution. To check whether the flux can be tolerated a similar approach to the one described by Vant-Hull (2002) is used. In the model a look-up table is included in which any flux limits

$$
A F D_{i}=f\left(T_{\mathrm{S}, i}, \dot{m}\right)
$$

can be used. In case of Solar Two both effects limiting the flux, the film temperature and the stresses in the tube, were transformed into a formulation for the $A F D$ in the form of equation 5. In the presented model we have implemented an integrated approach calculating the film temperature. The approach described above is fast, but it calculates only one single temperature for the front side element, which represents a mean temperature. In reality, the temperature of the inner and outer tube surface changes from the tube crown to the sides. For energetic calculations the usage of a mean temperature can be appropriate. However, for the limiting film temperature the highest temperature is relevant which cannot be calculated by the described approach. To estimate the highest temperature at the crown of the tube we can use the assumption that the flux density on the outer tube surface is almost symmetric in circumferential direction and therefore $\partial T / \partial \phi \approx 0$. As a result the flux hitting the crown has to be transported through the tube radially and the crown temperature $T_{\mathrm{Fc}}$, which is the highest temperature, can be calculated by solving the equation

$$
\begin{gathered}
\alpha \dot{q}_{\mathrm{F}}^{\prime \prime}-\epsilon \sigma\left(T_{\mathrm{Fc}}^{4}-T_{\infty}^{4}\right)-h_{\mathrm{conv}}\left(T_{\mathrm{Fc}}-T_{\infty}\right)= \\
\frac{1}{\frac{d_{\mathrm{O}}-d_{\mathrm{I}}}{2 k}+\frac{1}{h}}\left(T_{\mathrm{Fc}}-T_{\mathrm{S}, i}\right) .
\end{gathered}
$$

As the heat transfer coefficient $h$ and the temperature of the salt $T_{\mathrm{S}}$ is known, the equation can be solved directly. Then the crown film temperature $T_{\text {Ic }}$ can be calculated by solving

$$
\begin{gathered}
\alpha \dot{q}_{\mathrm{F}}^{\prime \prime}-\epsilon \sigma\left(T_{\mathrm{Fc}}^{4}-T_{\infty}^{4}\right)-h_{\mathrm{conv}}\left(T_{\mathrm{Fc}}-T_{\infty}\right)= \\
h\left(T_{\mathrm{Ic}}-T_{\mathrm{S}, i}\right) .
\end{gathered}
$$


The thermal model delivers different values representing the output of the receiver which can be maximized, e.g. thermal output or intercepted power. In case of a violation of the AFD and/or the maximum salt temperature the exceedance

$$
e=\max \left(\max \left(\xi_{i}-\xi_{\text {lim }}\right), 0\right) .
$$

In the equation $\xi$ can be the flux or the maximum film temperature. The exceedance is used to calculated a penalty factor

$$
p=\exp (-c \cdot e)
$$

with a user defined constant $c>1$. This penalty factor $0<p<1$ is then applied to make the output values in case of a violation unattractive.

\section{Validation of the thermal model}

In order to proof its validity the results of the model are compared to those of a much more detailed model. In order to validate the accelerated receiver model, its results are compared to a high detail model. In this study, the simplified model is compared to the ASTRID C model (Frantz et al. (2016)). ASTRID @ is a thermal FEM model, which considers both the absorber tubes and the insulation. The local solar irradiation coming from the heliostat field is simulated by the raytracing software SPRAY and is applied as a boundary condition. The heat transfer to the fluid is modeled using onedimensional fluid flow elements allowing mass and heat transportation. The local heat transfer coefficients are computed based on Nusselt correlations as a function of the local fluid temperature and Reynolds number. The thermal radiative exchange between the absorber tubes and the insulation as well as the radiation to the ambient is modeled using the radiosity method. The natural and forced convection losses are modeled by local heat transfer coefficients issued from CFD simulations. A mass flow control algorithm adapts the mass flow iteratively for each flow path through the receiver in order to get the desired outlet temperature for different load scenarios..

Both models were used to simulate the thermal behavior of the Solar Two power plant. From the detailed flux density distribution over the circumference of the tube issued from the ASTRID (C) model a flux density distribution with just one value per tube in horizontal direction was calculated. The resulting integral values of both models are given in table 1. Additionally, experimental results of the Solar Two power plant are listed. Both models 
Table 1: Comparison of the integral results of both models in the design point (incident power $48 \mathrm{MW}$ ). Comparison values of the Solar Two plant are given as well (Pacheco et al. (2002)).

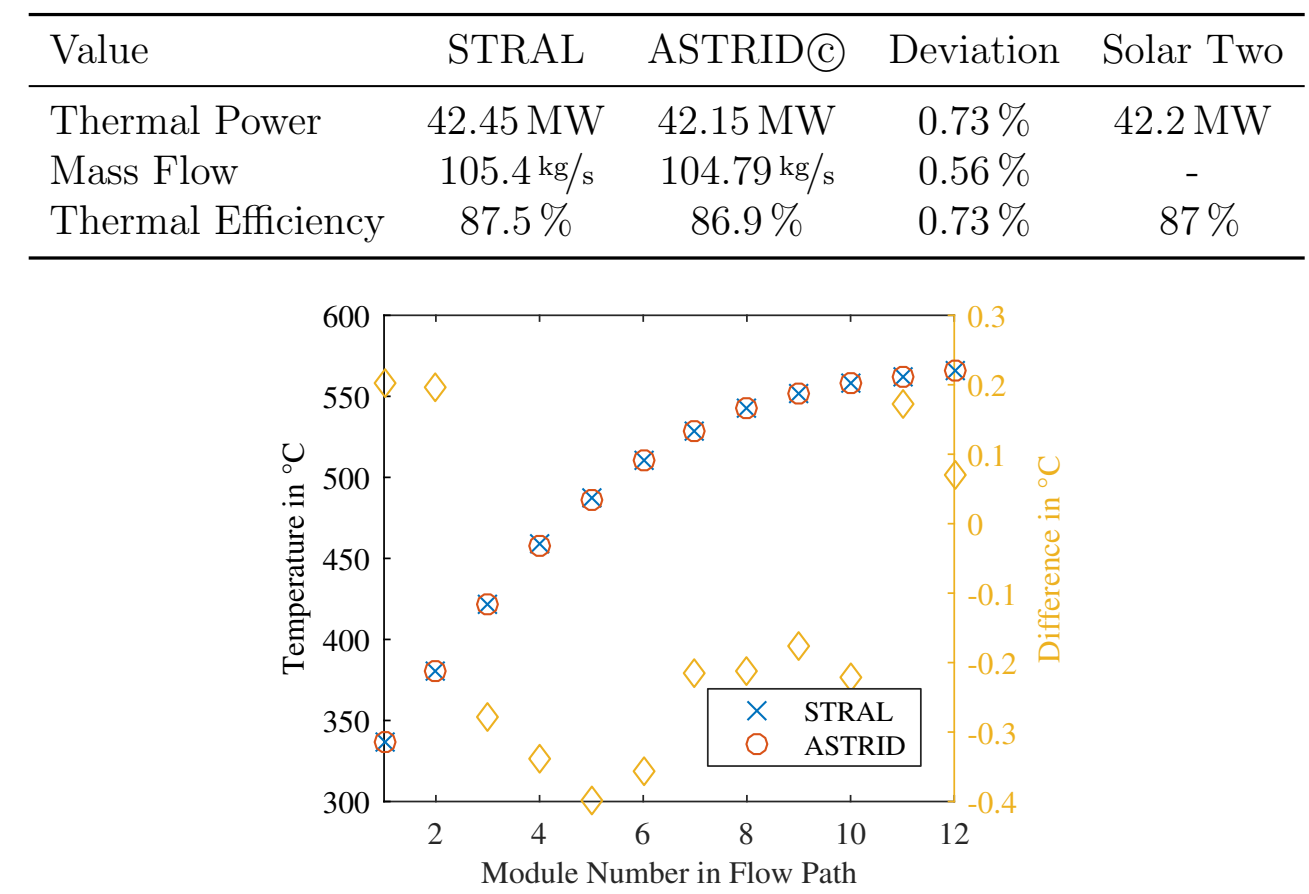

Figure 4: Maximum fluid temperature for each module in the two flow paths

give almost the same results and the values are similar to those reported for the real Solar Two Plant.

As described in section 3.2.2 the AFD is calculated based on mass flow and local temperature. Therefore, in addition to the mass flow the temperature distribution in the receiver is of particular interest. The highest fluid temperature for each panel is shown in Fig. 4. The fluid temperature rises from approximately $340^{\circ} \mathrm{C}$ behind the first panels to the design outlet temperature $565^{\circ} \mathrm{C}$ behind the last panel. A slight difference between the two models can be observed, but overall the temperature distributions are in good agreement. Consequently, the model can be used to calculated the local allowable flux density based on mass flow and fluid temperature.

Beside the limit based on the AFD the model is capable to calculate the crown film temperature which poses an additional limit for a safe operation of the plant. To validate the calculation approach for the crown film tem- 


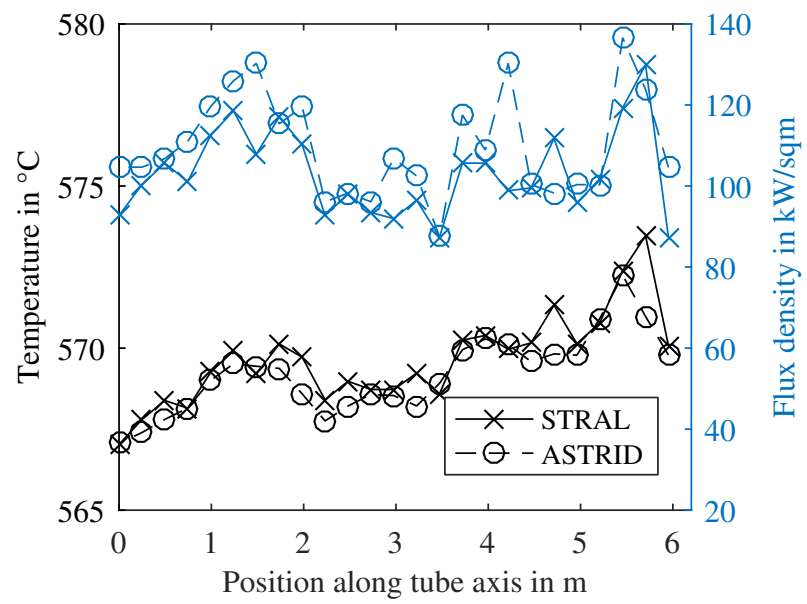

Figure 5: Comparison of the peak film temperature in the tube with the highest film temperatures.

perature the values for $T_{\text {Ic }}$ calculated by the STRAL model are compared to the highest film temperature calculated by the ASTRID(c) model. Figure 5 shows the comparison of the peak film temperature of a single tube as function of the position. Additionally, the flux density used in both modes is presented as well. In case of the ASTRID (C) model the highest flux density of all circumferential elements at the given axial position is used, as the film temperature is expected to correlate with the peak flux. The temperature distribution calculated by the STRAL model matches the temperatures simulated by the ASTRID@ model quite well. The highest differences occur in positions where the flux differ as well, which can be explained by the necessary averaging procedure for the flux. But the highest difference is smaller than $2{ }^{\circ} \mathrm{C}$ and therefore acceptable.

Altogether, the validation has proven that the model is accurate enough to be used for calculation of the AFD and the film crown temperature.

\section{Case Definition}

For the demonstration of the capabilities of the aim point optimization a new field layout and receiver is designed. It would have been preferable to demonstrate the capabilities for an existing plant, but in most cases the required detailed information on the design are not publicly available. The Solar Two plant, of which design is documented very well, is too small compared to the plants built nowadays. 
Table 2: Summary of properties of the used heliostat design

\begin{tabular}{lc}
\hline Total reflective area & $121 \mathrm{~m}^{2}$ \\
Width & $12.93 \mathrm{~m}$ \\
Height & $9.57 \mathrm{~m}$ \\
Pedestal height & $5.2 \mathrm{~m}$ \\
Surface error & $1.3 \mathrm{mrad}$ \\
Tracking error & $0.65 \mathrm{mrad}$ \\
Total reflectivity & $90.24 \%$ \\
\hline
\end{tabular}

Table 3: Summary of the receiver parameter used for demonstration

\begin{tabular}{lc}
\hline Optical tower height & $190 \mathrm{~m}$ \\
Circumscribing diameter & $15.82 \mathrm{~m}$ \\
Radiated height & $20 \mathrm{~m}$ \\
Number of flow paths & 2 \\
Number of panels & 12 \\
Number of tubes per panel & 117 \\
Tube inner diameter & $30 \mathrm{~mm}$ \\
Tube outer diameter & $35 \mathrm{~mm}$ \\
Inlet temperature & $290^{\circ} \mathrm{C}$ \\
Outlet temperature & $565^{\circ} \mathrm{C}$ \\
\hline
\end{tabular}

The location of the Redstone thermal power plant $\left(28^{\circ} \mathrm{S}\right.$ and $\left.23^{\circ} \mathrm{E}\right)$ is chosen as position for the virtual plant. Heliostats similar to the Sanlucar 120 (Osuna et al. (2006)) are used. Their most relevant parameter are summarized in Tab. 2. A field layout is created using HFLCAL (Schwarzbözl et al. (2009)). The resulting layout with a total number of 6482 heliostats is shown in Fig. 6. As indicated by the different colors, 20 groups with different canting distances are used.

The receiver is designed with an approximated thermal output of $450 \mathrm{MW}$. Its parameters are summarized in Tab. 3. The salt enters the receiver in the southern part and flows through the panels as depicted in Fig. 7. The salt flow is crossed after the third panel. In the simulation not all tubes of each panel are resolved in order to reduce the computational effort. The simulations are performed using 5 representative tubes of the 117 tubes per panel and 25 elements per tube in lengthwise direction. This results in a 


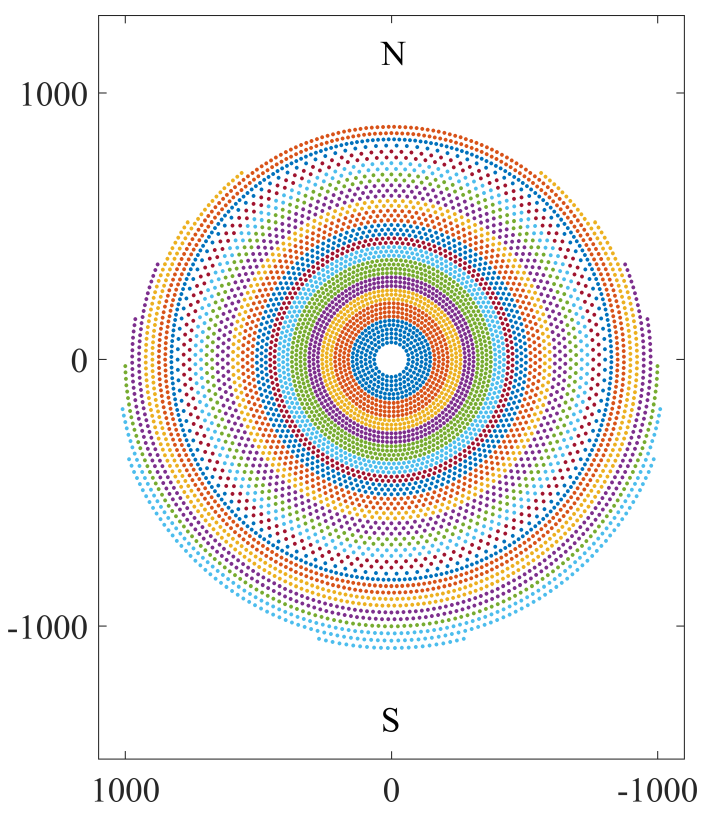

Figure 6: Field layout

total amount of 1500 tube elements for the whole receiver.

The lookup table for the allowable flux density is calculated using the formula taken from Vant-Hull (2002) from the bulk salt temperature in degree Fahrenheit $\theta$

$$
\begin{gathered}
A F D=\left(842.27-1.5514 \cdot \theta+4.613 \cdot 10^{-3} \cdot \theta^{2}\right. \\
\left.-3.2073 \cdot 10^{-6} \cdot \theta^{3}\right) \cdot\left(0.3+0.7 \cdot \frac{v}{v_{\mathrm{dp}}}\right) .
\end{gathered}
$$

This $A F D$ is applied to limit the net flux density

$$
\dot{q}_{\text {net }}^{\prime \prime}=\frac{\dot{Q}_{\text {cond }}}{A_{\mathrm{p}}} .
$$

For a simpler evaluation we define the load factor

$$
L F=\frac{\dot{q}_{\text {net }}^{\prime \prime}}{A F D}
$$

as the ratio of the local radiation divided by the local $A F D$. The second limit resulting from the temperature limit for the fluid is applied directly by comparing the crown film temperature to the limit of $600^{\circ} \mathrm{C}$. 


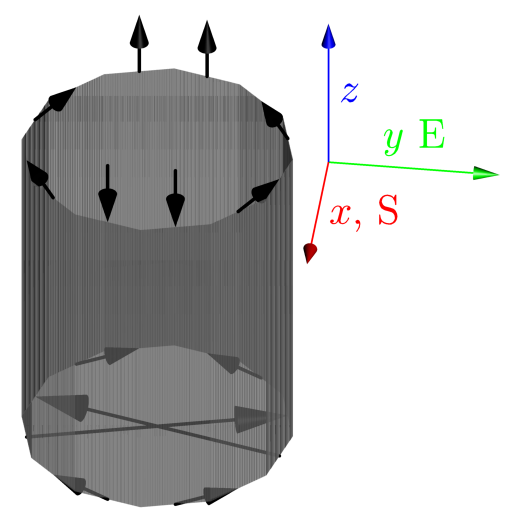

Figure 7: Visualization of the receiver and its flow pattern

All simulation are carried out for solar noon on the 21st of march, which will be referred as the design point.

\section{Results and Discussion}

\subsection{Parameter based aiming}

Firstly, we want to find a suitable choice of parameter which combines a flux which is within the limits and leads to a high intercept factor $I C$ defined as the ratio of the actual radiation which hits the receiver and the hypothetical amount of radiation hitting a receiver with infinite dimensions. A parameter variation is performed for the design point neglecting the tracking error. The results are shown in Fig. 8. In the plot the results for the choice of $\xi=0$ are shown as well, which corresponds to the original VantHull aiming. It is clearly observable that independent of the choice of $f_{0}$ the original Vant-Hull strategy does not create a flux distribution which is within the predefined limits. When extending the strategy with the second parameter one can see that high values for $f_{0}$ and $\xi$ are favorable for a high intercept factor $I C$. The highest film temperature decreases with increasing $\xi$ for the lower values of $f_{0}$. In case of the lower values for $f_{0}$ the load factor first decreases with increasing $\xi$ and after becoming minimal around $\xi \approx 2.25$ it increases again. For the highest $f_{0}$ shown in the plot the maximum load factor increases monotonically with increasing $\xi$. The parameter based aiming strategy barely creates suitable aim point distributions. The parameter 


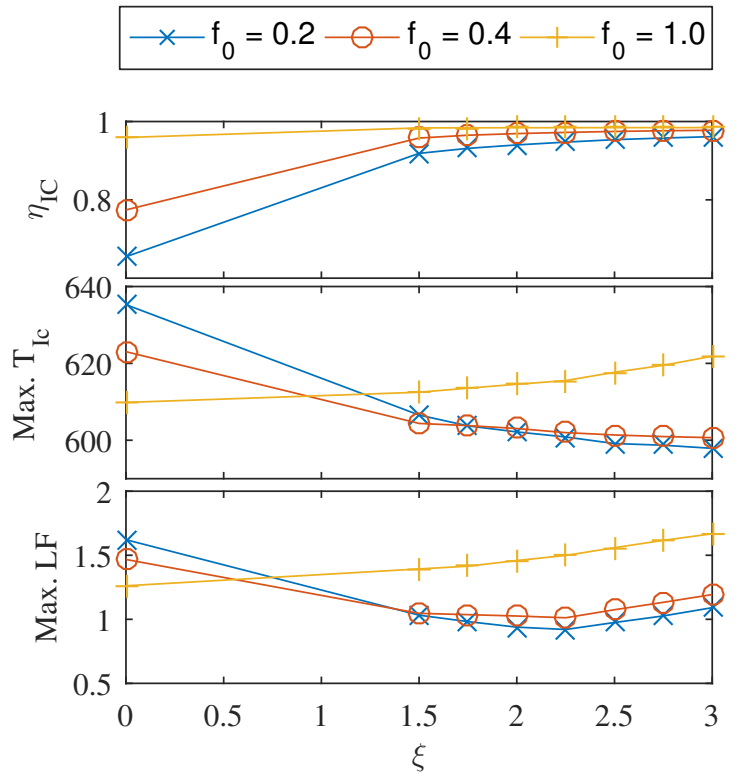

Figure 8: Results of the variation for the parameter based aiming strategy

combination $f_{0}=0.2$ and $\xi=2.5$ creates the best intercept with respect to all limits. This will be used as reference for the aim point optimization.

\subsection{Aim point optimization}

For a first demonstration the aim point optimization is performed for the design point. An aim point grid with 60 points in circumferential direction and 30 points in vertical direction is used. Two different strategies are compared: in the first strategy each heliostat is allowed to aim on the vertical axis of the cylinder. By this restriction the number of possible combination is limited which reduces the time required for the precalculation step. In the second strategy each heliostat has the degree of freedom to choose other aim points in circumferential direction. In both cases the optimization is initialized with the solution of the modified Vant-Hull aiming. It would have been possible to start from a random heliostat aim point assignment, but starting from a good valid solution greatly improves the performance and makes it easier to compare the results. For the optimization the thermal output of the receiver is chosen as objective value. The resulting progress of the optimization is shown in Fig. 9. In the initial phase of the optimization the output of the receiver increases sharply. In this phase the cylindric strategy outperforms the on-axis strategy. After the initial phase the progress 


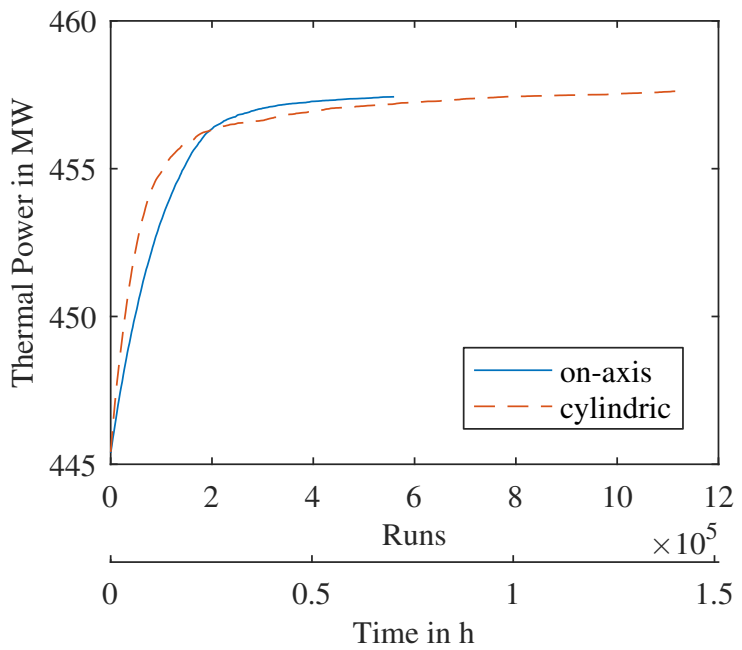

Figure 9: Improvement of the thermal power of the receiver during the optimization using the two different strategies.

of the optimization slows down. As this phase begins earlier for the cylindric strategy, the on-axis strategy produces an aim point assignment with a higher performance after approximately 200000 runs. In case of the on-axis strategy the optimization is stopped after 560000 runs as no significant improvement is obtained anymore. The optimization for the cylindric strategy is carried on for another 560000 runs. After these runs the optimization with the cylindric strategy results in an aim point assignment with a slightly higher performance. In Fig. 9 the duration of the optimization is given on the second $\mathrm{x}$-axis: the optimization was performed on a workstation computer with two Intel ${ }^{\circledR}$ Xeon ${ }^{\circledR}$ E5-2687W processors running at $3.1 \mathrm{GHz}$ using 30 parallel threads. On this machine one run takes less than $5 \mathrm{~ms}$. The initial phase with the sharp increase of the output takes less than 15 minutes.

The intercept factor and thermal output for the modified Vant-Hull aiming and the optimized aim point assignments with the two different strategies are summed up in Tab. 4 and compared to the results for an aiming strategy, in which all heliostats aim on the centerline of the receiver. This strategy maximizes the intercept factor, but the maximum temperature and fluxes are way beyond the limits. Nevertheless, this strategy gives an upper bound for the achievable intercept factor. Therefore, in table 4 the so-called aiming efficiency is given which is defined as the ratio of intercept factors of the used strategy and the central aiming strategy. All values are given for a cal- 
Table 4: Comparison of the key figures for the parameter based aiming and the optimized aim point assignments. As additional reference the results for a central strategy are given in which all heliostats aim on the centerline of the receiver. This aiming strategy does not produce a solution within the limits.

\begin{tabular}{|c|c|c|c|c|}
\hline & $\begin{array}{l}\text { Central } \\
\text { aiming }\end{array}$ & $\begin{array}{c}\text { Parameter } \\
\text { based aiming }\end{array}$ & $\begin{array}{l}\text { Optimized } \\
\text { on axis }\end{array}$ & $\begin{array}{l}\text { Optimized } \\
\text { cylindric }\end{array}$ \\
\hline$\dot{\leftrightarrows}$ Intercept factor & $98.5 \%$ & $95.4 \%$ & $97.8 \%$ & $97.8 \%$ \\
\hline $\begin{array}{l}\text { ¿ Thermal power } \\
0 \text { Th }\end{array}$ & $459 \mathrm{MW}$ & $445 \mathrm{MW}$ & $457 \mathrm{MW}$ & $458 \mathrm{MW}$ \\
\hline Aiming efficiency & $100 \%$ & $96.8 \%$ & $99.3 \%$ & $99.3 \%$ \\
\hline$\doteq$ Intercept factor & $98.2 \%$ & $94.7 \%$ & $97.3 \%$ & $97.3 \%$ \\
\hline (D) Thermal power & $458 \mathrm{MW}$ & $442 \mathrm{MW}$ & $455 \mathrm{MW}$ & $455 \mathrm{MW}$ \\
\hline A Aiming efficiency & $100 \%$ & $96.4 \%$ & $99.1 \%$ & $99.1 \%$ \\
\hline
\end{tabular}

culation neglecting the tracking error as it is done during the optimization and the mean values of five runs taking the tracking error into account. The aiming efficiency impressively underlines the capabilities of the aim point optimization: less than one percent of the power coming from the field are lost due to the distribution of the aim points in order to obtain a flux density distribution which is within the limits. It is very likely that the value obtained from the optimization is close to the global optimum.

When comparing the intercept factors of the different strategies with and without tracking error, we can see that the optimized aim point assignments are more robust to a performance loss caused by the inaccurate tracking, because the intercept factor is only reduced by $0.5 \%$ in the optimized cases instead of $0.7 \%$ in case of the parameter based aiming.

Figure 10 gives a deeper insight into the resulting flux density distribution and the crown film temperature for the optimized case with all heliostats aming on the axis.

In Fig. 10a the net absorbed flux density and its limit defined by Eq. 10 is shown. The different graphs correspond to the different calculated tubes in the flow path. The $A F D$ is increasing slightly in the first three panels due to the increasing temperature. After that it drops significantly to the outlet. For each panel the actual flux is at least at one position very close to the $A F D$. In the panels close to the outlet the flux reduces the closer the tubes are to the outlet, because the $A F D$ for the next panel is significantly lower and the flux has to change to the lower values. In the last panel the 


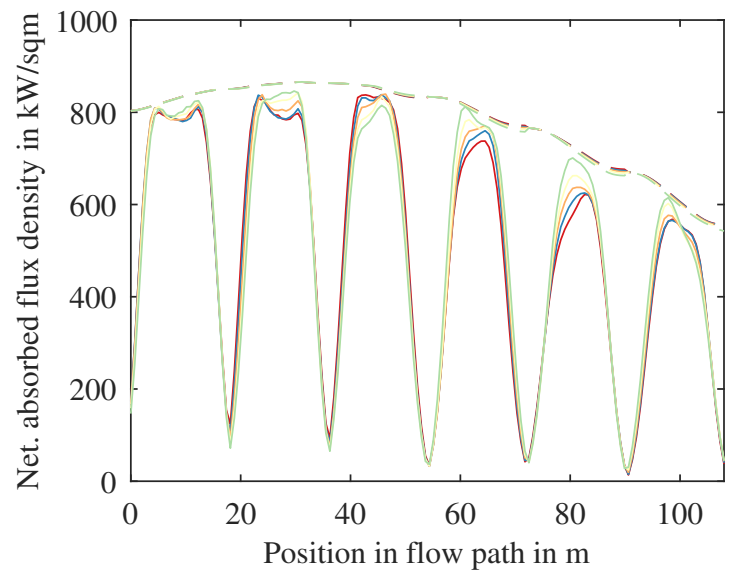

(a)

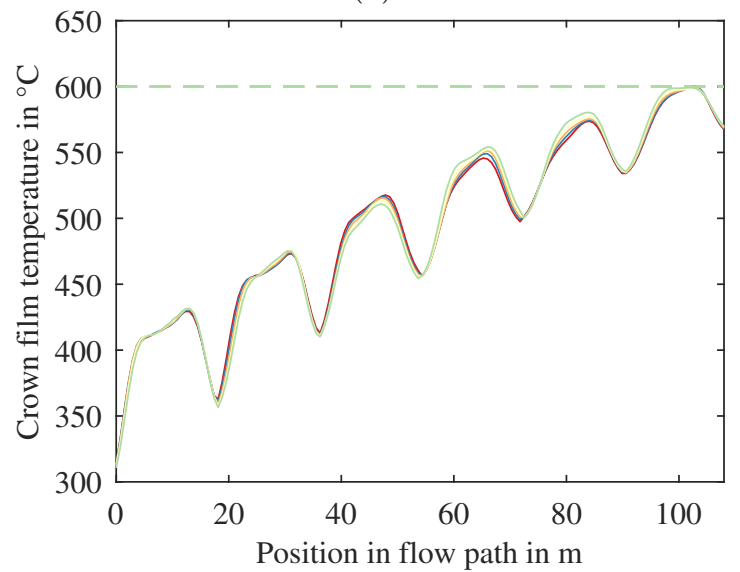

(b)

Figure 10: Net absorbed flux density and film temperature along the flow in one flow path. The different tubes shown are represented by different colors. The dashed line shows the individual limit for the corresponding tube. 


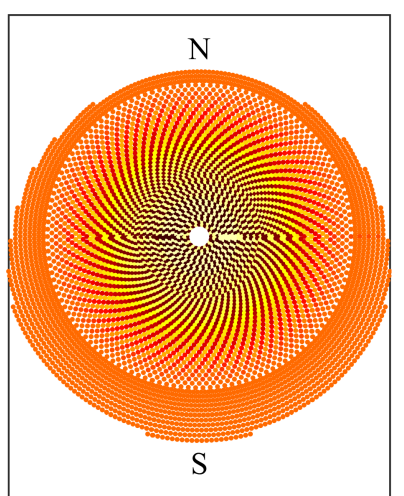

(a) Parameter based aiming

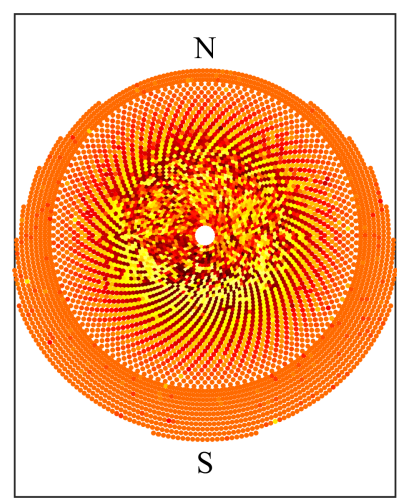

(b) Optimized - axis

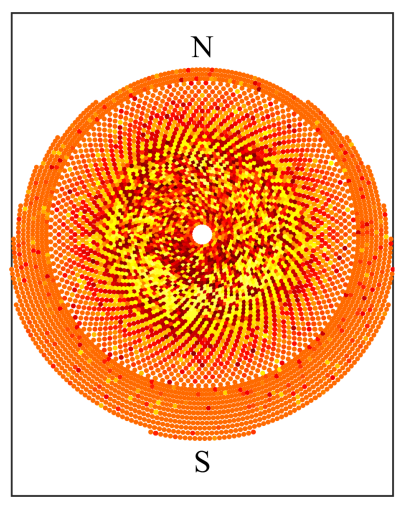

(c) Optimized - cylindric

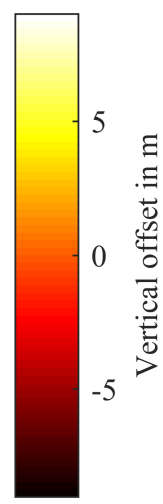

$$
\text { ic }
$$

Figure 11: Comparison of the vertical offset for the three analyzed aim point strategies

film temperature limit becomes the limiting factor (see Fig. 10b).

Figure 11 gives an overview about the vertical displacement of the aim point of each heliostat compared to the centerline of the receiver. In case of the parameter based aiming (Fig. 11a) the vertical offset of the heliostats follows a regular pattern: neighboring heliostats aim to the lower part and the upper part of the receiver respectively and with increasing distance the vertical displacement becomes zero. For both optimized aim point assignments the vertical displacement becomes zero with increasing distance with some exceptions. The regular pattern of the neighboring heliostats has vanished in the central part of the field in both optimized cases. The mean vertical displacement with a value of $2.3 \mathrm{~m}$ is lower for both optimized cases compared to the parameter based aiming with a mean vertical displacement of $3.2 \mathrm{~m}$. The lower displacement explains the higher intercept factor and the higher robustness of the aiming to the tracking error: the closer the heliostat aims to the centerline the higher the inaccurate tracking has to become to lead to an increasing spillage. The horizontal shift of the aim points along the cylindric surface is shown in Fig. 12. This shift follows a rather regular pattern: the heliostats close to the tower are shifted towards the outlet side of the receiver. But the performance increase due to that shift is almost negligible in the presented demonstration case. In case of receiver with a lower ratio of height to diameter this shift in circumferential direction might become more important. 

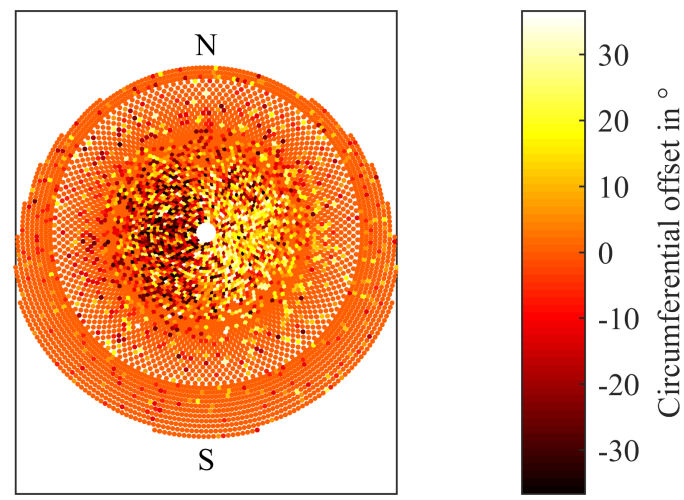

Figure 12: Displacement of the aim points along the circumference of the cylinder.

\section{Conclusion and Outlook}

A fast thermal model for external receivers with molten salt is developed and presented. The model calculates mass flow and local temperatures of the salt and tube walls. It is validated with an ANSYS based model (ASTRIDC) and the accuracy of the results is proven sufficient. This thermal model is integrated in a ray tracing based aim point optimization procedure using an ant colony optimization approach. In the optimization the thermal output of the receiver is maximized while considering both flux density limits calculated from mass flow and local salt temperature and an additional limit for the crown film temperature. The capabilities of the aim point optimization are demonstrated for a hypothetical power tower with a thermal power of $450 \mathrm{MW}$. Two different optimization strategies are analyzed: firstly, a strategy in which the heliostats had only the degree of freedom to aim on the vertical axis of the cylinder and one in which they can aim freely on the cylinder surface. The results are compared to the newly developed modified Vant-Hull strategy and the hypothetical limit where all heliostats aim on the receiver centerline. On a state-of-the-art workstation an improvement of more than $2 \%$ points was obtained within an optimization time of $15 \mathrm{~min}$. In contrast to other proposed strategies the optimization procedure is not restricted to strategies in which the heliostats aim on the vertical axis of the cylinder: on the contrary, this method is capable to generate heliostat aim point assignment where the helisotats aim on any point on the receiver surface. In the specific analyzed case this unrestricted strategy did not lead to an improved performance.

In the future it would be interesting to use the system to generate aim 
point assignments for an existing plant to analyze the improvements which can be realized by an optimized aim point distribution under more realistic constraints. The optimization strategy could be integrated as well in a control system of the power plant as it has already been demonstrated in a power tower with an open volumetric receiver (Schwarzbözl et al. (2016)). Such a system could generate new aim points distribution within a given intervall of several minutes to adapt the aim points to the given conditions. As a vision the system could even react to clouds interrupting the normal operation if combined with a nowcasting system.

\section{Acknowledgment}

This work was carried out with financial support from the German Ministry for Economic Affairs and Energy on the basis of a decision by the German Parliament.

Belhomme, B., Pitz-Paal, R., Schwarzbözl, P.. Optimization of heliostat aim point selection for central receiver systems based on the ant colony optimization metaheuristic. Journal of Solar Energy Engineering, Transactions of the ASME 2014;136(1). Cited By :7 Export Date: 31 January 2017.

Belhomme, B., Pitz-Paal, R., Schwarzbözl, P., Ulmer, S.. A new fast ray tracing tool for high-precision simulation of heliostat fields. Journal of Solar Energy Engineering, Transactions of the ASME 2009;131(3):03100210310028. Cited By :37 Export Date: 31 January 2017.

Besarati, S.M., Yogi Goswami, D., Stefanakos, E.K.. Optimal heliostat aiming strategy for uniform distribution of heat flux on the receiver of a solar power tower plant. Energy Conversion and Management 2014;84:234243.

Frantz, C., Fritsch, A., Uhlig, R.. ASTRID Advanced Solar Tubular ReceIver Design: A Powerful Tool for Receiver Design and Optimization; Abu Dhabi.

García-Martín, F.J., Berenguel, M., Valverde, A., Camacho, E.F.. Heuristic knowledge-based heliostat field control for the optimization of the temperature distribution in a volumetric receiver. Solar Energy 1999;66(5):355-369. 
Osuna, R., Olavarra, R., Morillo, R., Sanchez, M., Cantero, F., FernandezQuero, V., Robles, P., Lpez, T., Esteban, A., Cron, F.. Ps10, construction of a 11mw solar thermal tower plant in seville, spain. In: Solar-PACES Conference, Seville, Spain, June. 2006. p. 20-23.

Pacheco, J.E., Bradshaw, R., Dawson, D., De la Rosa, W., Gilbert, R., Goods, S., Hale, M., Jacobs, P., Jones, S., Kolb, G.. Final test and evaluation results from the solar two project. Report No SAND2002-0120, Sandia National Laboratories, Albuquerque, NM 2002;45.

Salom, A., Chhel, F., Flamant, G., Ferrire, A., Thiery, F.. Control of the flux distribution on a solar tower receiver using an optimized aiming point strategy: Application to themis solar tower. Solar Energy 2013;94:352-366.

Sanchez-Gonzalez, A., Rodriguez-Sanchez, M.R., Santana, D.. Aiming strategy model based on allowable flux densities for molten salt central receivers. Solar Energy 2016;.

Schwarzbözl, P., Pitz-Paal, R., Schmitz, M.. Visual hflcal-a software tool for layout and optimisation of heliostat fields. In: Proceedings. 2009. p. 8.

Schwarzbözl, P., Rong, A., Macke, A., Sck, J.P., Ulmer, S.. An automated model-based aim point distribution system for solar towers. AIP Conference Proceedings 2016;1734(1):020023.

Vant-Hull, L.L.. The role of allowable flux density in the design and operation of molten-salt solar central receivers. Journal of Solar Energy Engineering 2002;124(2):165-169. 10.1115/1.1464124. 